\title{
Questioning the existence of a unique ground-state structure for Si clusters
}

\author{
Waldemar Hellmann, ${ }^{1}$ R. G. Hennig, ${ }^{2}$ Stefan Goedecker, ${ }^{1}$ C. J. Umrigar, ${ }^{3}$ Bernard Delley, ${ }^{4}$ and T. Lenosky ${ }^{5}$ \\ ${ }^{1}$ Institut für Physik, Universität Basel, CH-4056 Basel, Switzerland \\ ${ }^{2}$ Department of Materials Science and Engineering, Cornell University, Ithaca, New York 14853, USA \\ ${ }^{3}$ Cornell Theory Center, Cornell University, Ithaca, New York 14853, USA \\ ${ }^{4}$ Paul Scherrer Insitut, WHGA/123, CH-5232 Villigen PSI, Switzerland \\ ${ }^{5}$ Department of Materials Science and Engineering, Ohio State University, Columbus, Ohio 43210, USA
}

(Received 21 November 2006; published 7 February 2007)

\begin{abstract}
Density functional and quantum Monte Carlo calculations challenge the existence of a unique ground-state structure for certain Si clusters. For Si clusters with more than a dozen atoms the lowest ten isomers are close in energy and for some clusters entropic effects change the energetic ordering of the configurations. Isotope pure configurations with rotational symmetry and symmetric configurations containing one additional isotope are disfavored by these effects. Comparisons with experiment are thus difficult since a mixture of configurations is to be expected at thermal equilibrium.
\end{abstract}

DOI: 10.1103/PhysRevB.75.085411

PACS number(s): 61.46.Bc, 71.20.Mq, 71.15.Mb, 05.10.Ln

\section{INTRODUCTION}

The determination of the structure of clusters is a difficult task. The standard experimental techniques such as x-ray diffraction and NMR methods that allow one to determine the atomic positions in crystals and molecules are not applicable to clusters. ${ }^{1}$ The main source of experimental information, ion mobility measurements, ${ }^{2}$ gives only crude information about the overall shape of a cluster. The exact atomic positions of all the atoms forming the cluster remain unknown. For this reason computational simulations provide a viable alternative to the experimental approach, which has been widely used for silicon clusters. From the theoretical point of view the ground-state structure of a solid state system is determined by the global minimum of the BornOppenheimer potential energy surface. Finding the global minimum requires global optimization algorithms. Two problems arise in this context. First, most global optimization algorithms give no guarantee for finding the global minimum within a finite amount of computer time. Second, the Born-Oppenheimer energy surface has to be calculated with very high precision.

Concerning the first point there is now a large amount of agreement between different methods for medium size clusters containing up to 19 atoms. ${ }^{3,4}$ Genetic algorithms, ${ }^{5-7}$ the big-bang method, ${ }^{8}$ the basin hopping method, ${ }^{9-11}$ and the minima hopping method ${ }^{12}$ give typically similar or even identical results. The discrepancies are rather due to different exchange-correlation functionals in different investigations. ${ }^{13}$

The existence of a well-defined ground-state structure is generally taken to be granted for silicon clusters. Silicon clusters are, however, very different from bulk silicon where the second lowest configuration (a fourfold coordinated defect $^{14}$ ) is $2.4 \mathrm{eV}$ higher than the crystalline ground state. Clusters are frustrated systems, where most of the atoms cannot adopt their favorite fourfold coordination. ${ }^{1}$ This can lead to small energy differences between different configurations. The significant deviations of the clusters bond lengths from the crystalline bond lengths shown in Fig. 1 illustrate this frustration.

\section{COMPUTATIONAL APPROACH}

In this work we extend the search for the ground-state configurations of silicon clusters $\mathrm{Si}_{n}$ in the range $7 \leqslant n \leqslant 19$ to a large number of low energy excited configurations. This is possible with the dual minima hopping method (DMHM) ${ }^{15}$ which has the property that it explores successively higher energy configurations after having found the global minimum.

Density functional theory (DFT) and quantum Monte Carlo theory are used to determine the energies of the clusters. The DFT calculations are performed using GAUSSIAN ${ }^{16}$ $\mathrm{DMol}^{3},{ }^{17,18}$ and CPMD. ${ }^{19}$ Table I shows that the high quality basis sets that were used by DMOL, CPMD, and GAUSSIAN give slightly different answers and might change the energetic ordering. In addition, there is a significant dependence on the exchange-correlation functional as can be seen by comparing the energy ordering of the Perdew-Burke-Ernzerhof (PBE) ${ }^{21}$ and $\mathrm{B} 3 \mathrm{LYP}^{22}$ functionals.

In order to unambiguously determine the energetic ordering of the configurations we perform one of the most accu-

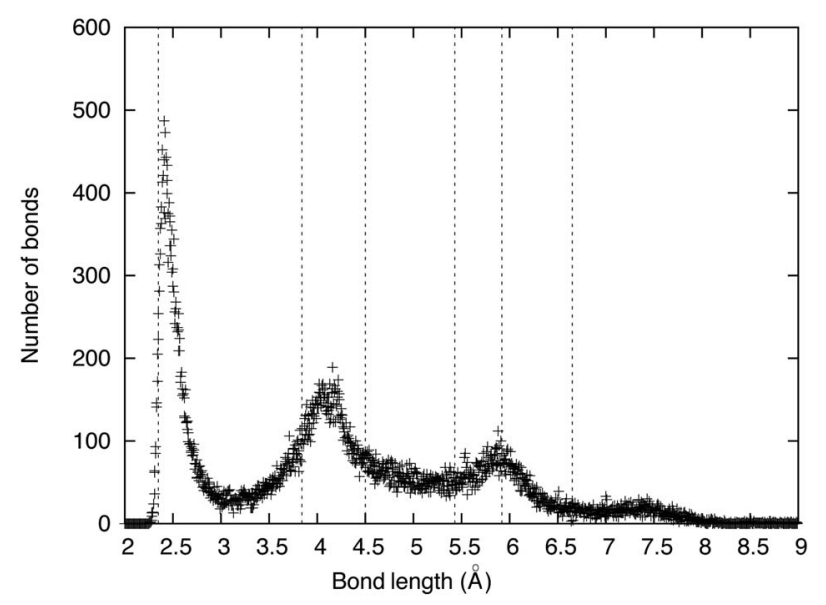

FIG. 1. The bond length distribution averaged over various lowlying $\mathrm{Si}_{17}$-configurations. The six vertical lines indicate the first to sixth nearest neighbor distances in the crystal. 
TABLE I. The energy differences in $\mathrm{mHa}$ between the low energy geometries $\mathrm{Si}_{13 a}, \mathrm{Si}_{13 b}, \mathrm{Si}_{13 c}, \mathrm{Si}_{13 d}, \mathrm{Si}_{13 e}, \mathrm{Si}_{13 f}, \mathrm{Si}_{16 a}, \mathrm{Si}_{16 b}$, $\mathrm{Si}_{17 a}, \mathrm{Si}_{17 b}, \mathrm{Si}_{18 a}$, and $\mathrm{Si}_{19 a}$ and the reference structures $\mathrm{Si}_{13}, \mathrm{Si}_{16}$, $\mathrm{Si}_{17}, \mathrm{Si}_{18}$, and $\mathrm{Si}_{19}$, proposed earlier as global minima in DFT. The GAUSSIAN (Ref. 16) calculations used the 6-311G(2d) basis and the DMol $^{3} 2005$ (Refs. 17 and 18) calculations the extended basis set. The CPMD (Ref. 19) calculations were performed with an accurate pseudopotential (Ref. 20) with a 35 Ry plane wave cutoff and a $30 \AA$ simulation cell.

\begin{tabular}{lccccc}
\hline \hline Cluster & $\begin{array}{c}\text { B3LYP } \\
\text { GAUSSIAN }\end{array}$ & $\begin{array}{c}\text { PBE } \\
\text { DMOL }\end{array}$ & $\begin{array}{c}\text { PBE } \\
\text { CPMD }\end{array}$ & $\begin{array}{c}\text { PBE } \\
\text { GAUSSIAN }\end{array}$ & DMC \\
\hline $\mathrm{Si}_{13}$ & 0.0 & 0.0 & 0.0 & 0.0 & 0.0 \\
$\mathrm{Si}_{13 a}$ & -11.7 & 6.3 & 7.1 & 5.3 & $-3.3 \pm 1.0$ \\
$\mathrm{Si}_{13 b}$ & -1.4 & 9.5 & 13.2 & 11.5 & $5.7 \pm 1.0$ \\
$\mathrm{Si}_{13 c}$ & 8.6 & 4.7 & 3.1 & 3.1 & $6.4 \pm 1.0$ \\
$\mathrm{Si}_{13 d}$ & -6.7 & -2.9 & 0.9 & 0.6 & $-5.7 \pm 1.1$ \\
$\mathrm{Si}_{13 e}$ & 3.6 & 3.9 & 4.1 & 4.2 & $6.1 \pm 1.0$ \\
$\mathrm{Si}_{13 f}$ & 4.2 & 7.0 & 8.5 & 8.0 & $10.6 \pm 1.0$ \\
$\mathrm{Si}_{13 g}$ & -6.3 & -0.8 & 2.6 & 2.3 & $0.0 \pm 1.1$ \\
$\mathrm{Si}_{16}$ & 0.0 & 0.0 & 0.0 & 0.0 & 0.0 \\
$\mathrm{Si}_{16 a}$ & 24.0 & -0.2 & -6.0 & -3.7 & $9.9 \pm 1.4$ \\
$\mathrm{Si}_{16 b}$ & 9.5 & 0.9 & 0.9 & 1.4 & $8.3 \pm 1.4$ \\
$\mathrm{Si}_{17}$ & 0.0 & 0.0 & 0.0 & 0.0 & 0.0 \\
$\mathrm{Si}_{17 a}$ & 7.4 & 8.4 & 2.6 & 2.7 & $6.3 \pm 1.5$ \\
$\mathrm{Si}_{17 b}$ & 10.2 & 11.6 & 8.4 & 8.4 & $13.2 \pm 1.7$ \\
$\mathrm{Si}_{18}$ & 0.0 & 0.0 & 0.0 & 0.0 & 0.0 \\
$\mathrm{Si}_{18 a}$ & 31.3 & 17.1 & 10.0 & 10.2 & $23.9 \pm 1.6$ \\
$\mathrm{Si}_{19}$ & 0.0 & 0.0 & 0.0 & 0.0 & 0.0 \\
$\mathrm{Si}_{19 a}$ & 15.4 & -1.0 & -4.2 & -1.9 & $2.1 \pm 1.7$ \\
\hline \hline & & & & & \\
\hline
\end{tabular}

rate electronic structure calculations feasible for these systems, namely quantum Monte Carlo (QMC) simulations. The QMC calculations are performed using the CHAMP code developed by Umrigar and Filippi. The $1 s, 2 s$, and $2 p$ electrons of $\mathrm{Si}$ are eliminated using a relativistic Hartree-Fock pseudopotential. ${ }^{23}$ A Slater-Jastrow type wave function is used as the trial wave function. The orbitals of the Slater determinant are taken from a DFT calculation with the GAMESS $^{24}$ code using the B3LYP functional. The parameters of the Jastrow function describing electron-electron, electron-nuclear, and electron-electron-nuclear correlations are optimized in variational Monte Carlo simulations using energy minimization. ${ }^{25}$ The final energies presented in Table I were obtained with the diffusion Monte Carlo (DMC) method.

\section{CONFIGURATIONAL ENERGIES}

Figure 2 shows the DFT energies of the ten lowest configurations of Si clusters containing 7-19 atoms. The energy difference between the global minimum and the second lowest minimum is $0.8 \mathrm{mHa}$ for $\mathrm{Si}_{11}, 0.9 \mathrm{mHa}$ for $\mathrm{Si}_{13}, 2.1 \mathrm{mHa}$ for $\mathrm{Si}_{14}, 3.1 \mathrm{mHa}$ for $\mathrm{Si}_{17}$, and $3.2 \mathrm{mHa}$ for $\mathrm{Si}_{19}$. For both $\mathrm{Si}_{13}$ and $\mathrm{Si}_{17}$ the ten lowest configurations are in an interval of about $10 \mathrm{mHa}$. Since room temperature corresponds to

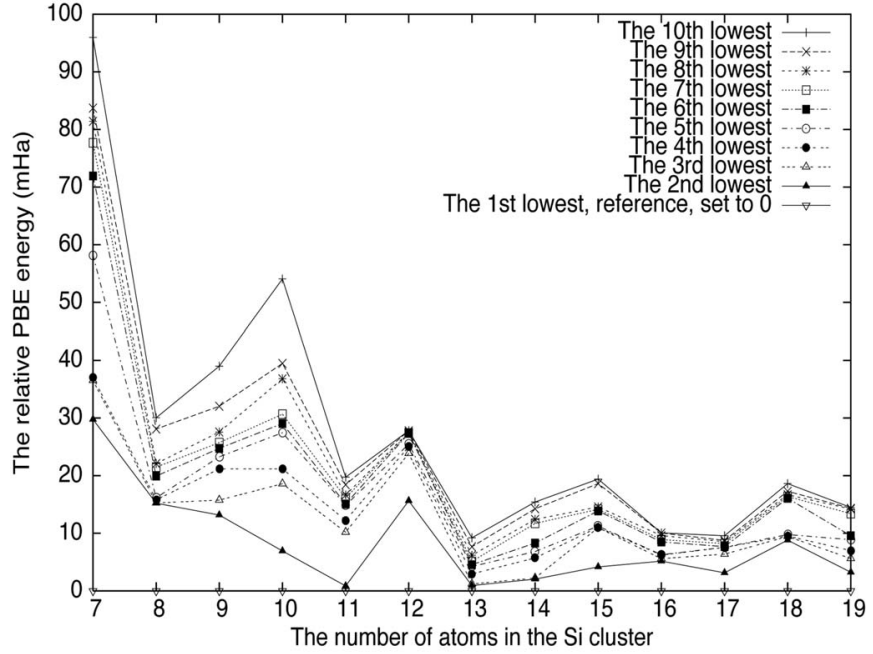

FIG. 2. The dependence of the PBE energy interval for the lowest ten configurations on the cluster size.

about $1 \mathrm{mHa}$, entropic effects play an important role for these clusters. The results of Fig. 2 are obtained with the $\mathrm{PBE}^{21}$ functional. Even though this functional is considered to be among the most accurate ones, its accuracy is clearly less than $1 \mathrm{mHa}$ which is the accuracy required to determine unambiguously the energetic ordering of the configurations. We perform QMC calculations for the clusters in order to determine accurately the energetic ordering.

Table I compares the QMC energy to the B3LYP and PBE energies. Figure 3 illustrates the corresponding configurations. The statistical error of the QMC calculations of about $1 \mathrm{mHa}$ is just sufficient to determine the energy ordering. QMC predicts a different energy ordering from either DFT functional, but the central feature remains that many configurations are nearly degenerate.

The new low-lying structures $\mathrm{Si}_{16 a}, \mathrm{Si}_{16 b}, \mathrm{Si}_{17 a}, \mathrm{Si}_{17 b}$, $\mathrm{Si}_{18 a}$, and $\mathrm{Si}_{19 a}$ which were found with DMHM and the reference structures $\mathrm{Si}_{16},{ }^{11} \mathrm{Si}_{17},{ }^{6} \mathrm{Si}_{18},{ }^{6}$ and $\mathrm{Si}_{19}$ (Ref. 7) were already presented in Ref. 15 . The structure $\mathrm{Si}_{13}$ was found by $\mathrm{Ho},{ }^{6}$ the rotationally symmetric $\mathrm{Si}_{13 d}$ structure was recently proposed by Hartke, ${ }^{26} \mathrm{Si}_{13 f}$ by Röthlisberger, ${ }^{27}$ and $\mathrm{Si}_{13 g}$ by Jeong et al. ${ }^{28}$ Using $\mathrm{DMHM}^{15}$ we have found low-lying structures $\mathrm{Si}_{13 a}, \mathrm{Si}_{13 b}, \mathrm{Si}_{13 c}$, and $\mathrm{Si}_{13 e}$. From the QMC results in Table I we conclude that the $\mathrm{Si}_{13 d}$ and $\mathrm{Si}_{13 a}$ configurations are the lowest energy structures. The $\mathrm{Si}_{13 a}$ structure found with DMHM contains the stable $\mathrm{Si}_{6}$ subunit. ${ }^{29}$ Besides, as one can see from the QMC results in Table I the structures $\mathrm{Si}_{16 a}$ and $\mathrm{Si}_{19 a}$ that contain the particularly stable tricapped trigonal prism (TTP) subunit are not energetically favored as one might expect.

\section{FINITE TEMPERATURE EFFECTS}

The DFT and QMC energies of the clusters are sufficiently close that zero-point energy and temperature can affect the energy ordering. For the $\mathrm{Si}_{13}, \mathrm{Si}_{13 a}$, and $\mathrm{Si}_{13 d}$ clusters we have zero point energies of 24.0, 25.0, and $24.5 \mathrm{mHa}$. For the $\mathrm{Si}_{19}$ and $\mathrm{Si}_{19 a}$ we have 38.8 and $38.0 \mathrm{mHa}$. So the differ- 

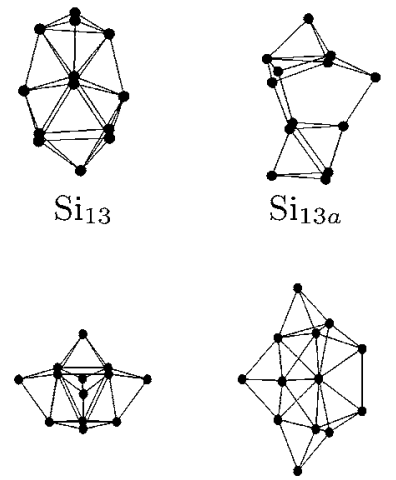

$\mathrm{Si}_{13 d}$

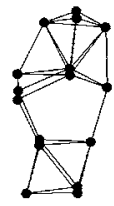

$\mathrm{Si}_{16}$
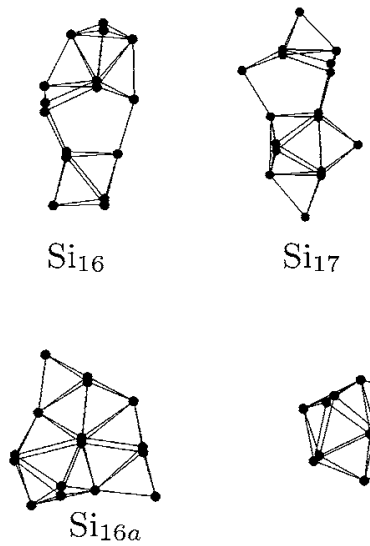

$\mathrm{Si}_{17}$

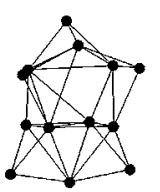

$\mathrm{Si}_{13 b}$

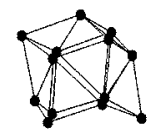

$\mathrm{Si}_{13 f}$

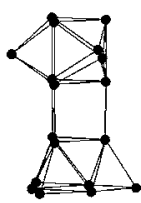

$\mathrm{Si}_{18}$
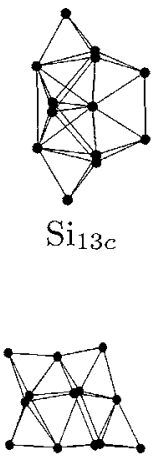

$\mathrm{Si}_{13 g}$

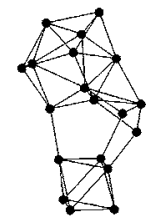

$\mathrm{Si}_{19}$
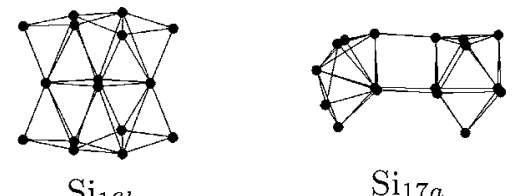

$\mathrm{Si}_{17 a}$
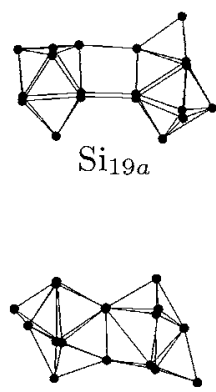

$\mathrm{Si}_{17 b}$

FIG. 3. Symmetrized geometries of low-lying $\mathrm{Si}_{13}, \mathrm{Si}_{16}, \mathrm{Si}_{17}$, $\mathrm{Si}_{18}$, and $\mathrm{Si}_{19}$ isomers.

ences of the zero point energies are all of the order of $\mathrm{mHa}$ and thus not negligible, but do not change the energetic ordering for the clusters we studied.

In order to study the entropic effects we calculated the rotational and vibrational free energy based on the harmonic frequencies obtained from density functional (PBE) calculations. ${ }^{30}$ The translational free energy does not depend on the configuration and was therefore not considered. If one compares the sum of the rotational and vibrational free energy for nonsymmetric configurations, one typically finds differences of about $0.5 \mathrm{mHa}$ at room temperature and about $1 \mathrm{mHa}$ close to the melting point of the clusters. ${ }^{31}$ This might change the energetic ordering, but we did not find a case where it actually does.

The situation is different if one compares a symmetric with a nonsymmetric configuration. Silicon occurs in nature mainly as a ${ }^{28} \mathrm{Si}$ or ${ }^{29} \mathrm{Si}$ isotope. The predominant isotope for silicon ${ }^{28} \mathrm{Si}$ (abundance $\sim 92 \%{ }^{32}$ ) has mass 28 and no nuclear spin, the ${ }^{29} \mathrm{Si}$ isotope (abundance $\sim 5 \%{ }^{32}$ ) has mass 29 and nuclear spin $1 / 2$. When studying configurations with rota-

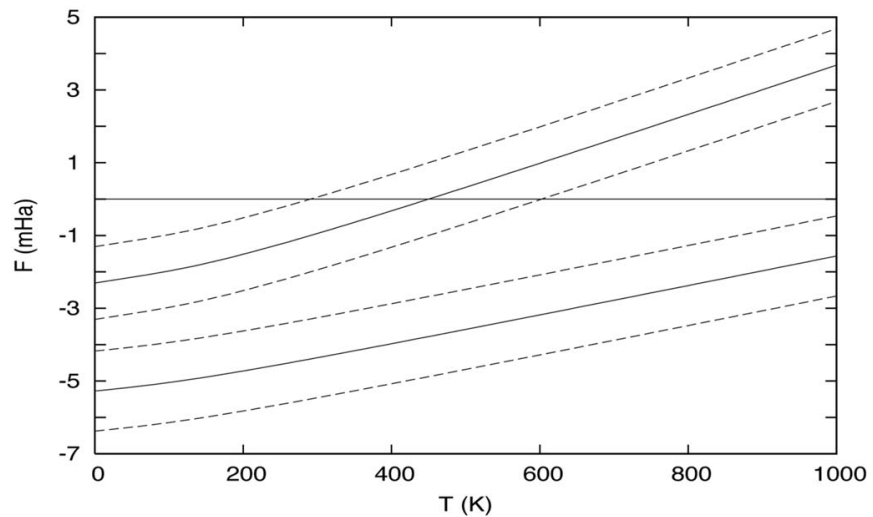

FIG. 4. The sum of the electronic (QMC, with errors), rotational, and vibrational (including zero point) free energy contributions for $\mathrm{Si}_{13 a}$ (upper band) and $\mathrm{Si}_{13 d}$ (lower band) configurations as a function of temperature with the $\mathrm{Si}_{13}$ free energy chosen as reference energy (solid line, set to 0 ). The bandlike structure indicated by the two dashed lines above and below each solid line represents the statistical error of the QMC results.

tional symmetry, we will consider pure clusters consisting only of ${ }^{28} \mathrm{Si}$ atoms since the presence of a ${ }^{29} \mathrm{Si}$ atom would destroy the rotational symmetry. One can easily estimate from the abundances of the isotopes that $\sim 34 \%$ of $\mathrm{Si}_{13}$ clusters will be pure clusters. For such a cluster with rotational symmetry, the order of the rotational subgroup enters into the formula for the rotational free energy. This leads to a weaker decrease of the free energy for symmetric configurations compared to nonsymmetric configurations and thus favors nonsymmetric structures. Centrifugal corrections of this treatment are usually of the order of some percent and can thus be neglected. In Fig. 4 we present the free energy curves for the structures $\mathrm{Si}_{13 a}$ and $\mathrm{Si}_{13 d}$ as a function of temperature with the $\mathrm{Si}_{13}$ free energy chosen as reference energy. The width of the bands for $\mathrm{Si}_{13 a}$ and $\mathrm{Si}_{13 d}$ represents the statistical errors in the QMC energies with respect to that of structure $\mathrm{Si}_{13}$. For the symmetric $\mathrm{Si}_{13 a}$ configuration the order of the rotational subgroup is 3 , for $\mathrm{Si}_{13 d}$ it is 2 , and for $\mathrm{Si}_{13}$ it is 1. This leads to a reversal of the energetic ordering of the structures $\mathrm{Si}_{13}$ and $\mathrm{Si}_{13 a}$ in the interval between 250 and $650 \mathrm{~K}$. Because of the entropic effect the $\mathrm{Si}_{13}$ configuration, which is the highest at zero temperature, becomes the lowest at temperatures above $1000 \mathrm{~K}$. At room temperature the $\mathrm{Si}_{13 a}$ and $\mathrm{Si}_{13 d}$ bands are separated by an energy gap in the range between $\sim 1.2$ and $\sim 5.5 \mathrm{mHa}$. This corresponds to a Boltzmann weight in the range between $0.7 \%$ and $30 \%$.

These considerations are only valid for clusters consisting purely of ${ }^{28} \mathrm{Si}$ atoms. The presence of a ${ }^{29} \mathrm{Si}$ isotope destroys the rotational symmetry. One can estimate from the abundances of the isotopes that $\sim 24 \%$ of $\mathrm{Si}_{13}$ clusters will contain one ${ }^{29} \mathrm{Si}$ isotope. If one ${ }^{28} \mathrm{Si}$ atom with nuclear spin 0 is replaced by a ${ }^{29} \mathrm{Si}$ isotope which has spin $1 / 2$, the nuclear wavefunction is a doublet and additional degeneracy comes from the fact that the isotope can replace any of the atoms. For a nonsymmetric cluster with $N$ atoms the degeneracy is thus $2 N$. For a symmetric cluster that has several equivalent atoms the degeneracy is, however, reduced. In the case of the $\mathrm{Si}_{13 a}$ structure there are, for instance, only five nonequivalent 


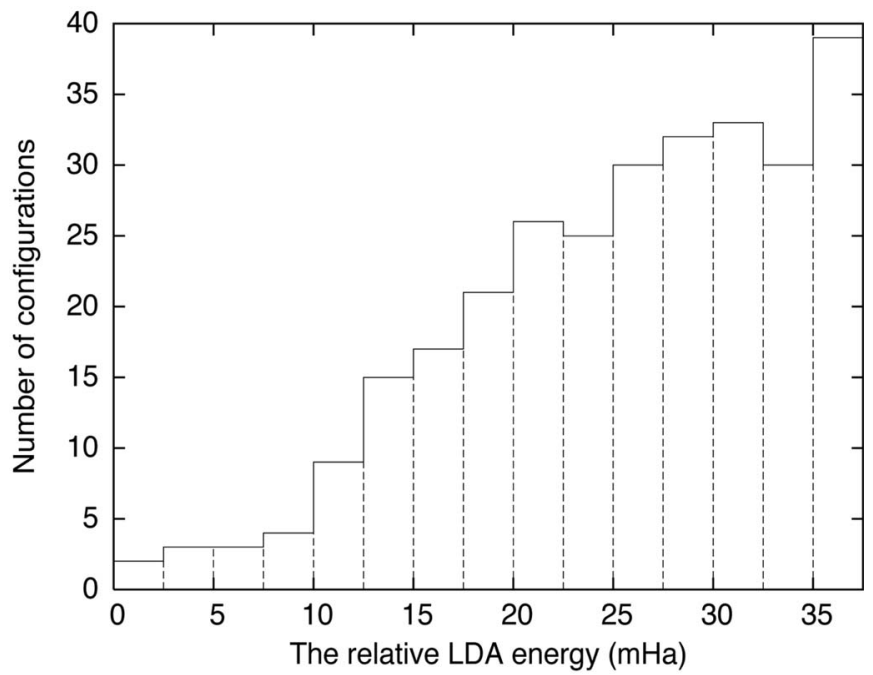

FIG. 5. The configurational density of states for $\mathrm{Si}_{17}$.

sites, $\mathrm{Si}_{13 d}$ has six and $\mathrm{Si}_{13}$ has nine. The nuclear entropy thus favors $\mathrm{Si}_{13}$ over $\mathrm{Si}_{13 d}$ by $-k T \ln \left(\frac{9}{6}\right)$ which is $\sim 0.4 \mathrm{mHa}$ at room temperature. In addition, the vibrational and rotational entropy contributions are slightly changed by the presence of an isotope leading to an effect of the same order of magnitude.

So far we have shown that the energetic spacing of the ten lowest structures decreases with cluster size. In addition, for a given cluster size the energetic spacing between configurations decreases with increasing energy. This is illustrated in Fig. 5 for $\mathrm{Si}_{17}$ clusters. The configurational density of states increases with energy.

\section{CONCLUSION}

In summary, we have shown that there exists a large number of configurations for certain silicon clusters that are energetically extremely close to the ground state. This feature was observed for $\mathrm{Si}_{13}$ and $\mathrm{Si}_{19}$ and it will presumably be even more important for larger cluster sizes that were not studied in this work. As a consequence, entropy effects that are usually neglected can change the energetic ordering of the lowest configurations. Entropy disfavors symmetric clusters $\mathrm{Si}_{n}$ in the range $13 \leqslant n \leqslant 19$ which contain in most cases no ${ }^{29} \mathrm{Si}$ isotope or one ${ }^{29} \mathrm{Si}$ isotope. Larger clusters will on average contain more than one ${ }^{29} \mathrm{Si}$ isotope and the symmetry related effects discussed above do not exist. However, for larger clusters the ten lowest configurations can be expected to lie within an even smaller interval. The entropic effects not related to symmetry considerations might thus easily change the energy ordering of clusters with more than 19 atoms. Even if there is no reordering, different structures can be so close in free energy that a mixture of two or more configurations will be found at thermal equilibrium. As a consequence measured properties of clusters can be some average of the properties of several low-lying isomers. Experimentally, Hudgins and Jarrold ${ }^{2}$ have confirmed the coexistence of different charged isomers at thermal equilibrium by performing high-resolution ion mobility measurements for medium-sized silicon cluster cations and anions. In particular, they have observed several peaks starting with $\mathrm{Si}_{17}{ }^{+}$ in the drift time distributions of silicon cluster cations. Since Rata et al. ${ }^{7}$ have found that the structures of lowest energy silicon cluster cations are essentially identical to those of lowest energy neutral clusters, we can expect the coexistence of different isomers of neutral clusters at thermal equilibrium. As for anions, Guliamov, Kronik, and Jackson ${ }^{33}$ have recently demonstrated that photoelectron spectroscopy (PES) can be used as a powerful probe of medium-sized silicon cluster anions that allows one to distinguish structurally distinct isomers. However, structurally similar isomers which will be likely present in an ensemble of medium-sized silicon cluster anions cannot be distinguished by the PES.

\section{ACKNOWLEDGMENTS}

We thank Markus Meuwly for discussions, X. C. Zeng and $\mathrm{A}$. Tekin for the $\mathrm{Si}_{13}$ cluster data, Matthias Krack for his help with Quickstep, the Swiss National Science Foundation for financial support, and the staff of the computing center at the University of Basel for technical support. The work at Cornell University was supported by NSF Grant No. EAR0530301 and NSF Grant No. DMR-0205328. Computational resources were provided by OSC, NERSC, and NCSA.
${ }^{1}$ B. Hartke, Angew. Chem., Int. Ed. 41, 1468 (2002).

${ }^{2}$ R. R. Hudgins, M. Imai, M. F. Jarrold, and P. Dugourd, J. Chem. Phys. 111, 7865 (1999).

${ }^{3}$ X. Zhu and X. C. Zeng, J. Chem. Phys. 118, 3558 (2003).

${ }^{4}$ X. Zhu, X. C. Zeng, Y. A. Lei, and B. Pan, J. Chem. Phys. 120, 8985 (2004).

${ }^{5}$ B. Hartke, J. Phys. Chem. 97, 9973 (1993).

${ }^{6}$ K.-M. Ho, A. A. Shvartsburg, B. Pan, Z.-Y. Lu, C.-Z. Wang, J. G. Wacker, J. L. Fye, and M. F. Jarrold, Nature (London) 392, 582 (1998).

${ }^{7}$ I. Rata, A. A. Shvartsburg, M. Horoi, T. Frauenheim, K. W. Michael Siu, and K. A. Jackson, Phys. Rev. Lett. 85, 546 (2000).
${ }^{8}$ K. A. Jackson, M. Horoi, I. Chaudhuri, T. Frauenheim, and A. A. Shvartsburg, Phys. Rev. Lett. 93, 013401 (2004).

${ }^{9}$ Z. Li and H. Scheraga, Proc. Natl. Acad. Sci. U.S.A. 84, 6611 (1987).

${ }^{10}$ J. P. K. Doye and D. J. Wales, Phys. Rev. Lett. 80, 1357 (1998).

${ }^{11}$ S. Yoo and X. C. Zeng, Angew. Chem., Int. Ed. 44, 1491 (2005).

${ }^{12}$ S. Goedecker, J. Chem. Phys. 120, 9911 (2004).

${ }^{13}$ S. Yoo and X. C. Zeng, J. Chem. Phys. 123, 164303 (2005).

${ }^{14} \mathrm{~S}$. Goedecker, T. Deutsch, and L. Billard, Phys. Rev. Lett. 88, 235501 (2002)

${ }^{15}$ S. Goedecker, W. Hellmann, and T. Lenosky, Phys. Rev. Lett. 95, 055501 (2005).

${ }^{16}$ GaUssian03, Revision B.01, M. J. Frisch, G. W. Trucks, H. B. 
Schlegel, G. E. Scuseria, M. A. Robb, J. R. Cheeseman, J. A. Montgomery, Jr., T. Vreven, et al., (Gaussian, Inc., Pittsburgh, PA, 2003).

${ }^{17}$ B. Delley, J. Chem. Phys. 92, 508 (1990).

${ }^{18}$ B. Delley, J. Chem. Phys. 113, 7756 (2000).

${ }^{19}$ CPMD Version 3.3: developed by J. Hutter, A. Alavi, T. Deutsch, M. Bernasconi, S. Goedecker, D. Marx, M. Tuckerman, and M. Parrinello (Max-Planck-Institut für Festkörperforschung and IBM Zürich Research Laboratory, 1995-1999).

${ }^{20}$ S. Goedecker, M. Teter, and J. Hutter, Phys. Rev. B 54, 1703 (1996)

${ }^{21}$ J. P. Perdew, K. Burke, and M. Ernzerhof, Phys. Rev. Lett. 77, 3865 (1996).

${ }^{22}$ J. P. Perdew, Phys. Rev. B 33, 8822 (1986); A. D. Becke, Phys. Rev. A 38, 3098 (1988); C. Lee, W. Yang, and R. G. Parr, Phys. Rev. B 37, 785 (1988); A. D. Becke, J. Chem. Phys. 98, 5648 (1993).

${ }^{23}$ J. R. Trail and R. J. Needs, J. Chem. Phys. 122, 174109 (2005).

${ }^{24} \mathrm{http} / / /$ www.msg.ameslab.gov/GAMESS/
${ }^{25}$ C. J. Umrigar and C. Filippi, Phys. Rev. Lett. 94, 150201 (2005).

${ }^{26}$ A. Tekin and B. Hartke, Phys. Chem. Chem. Phys. 6, 503 (2004).

${ }^{27}$ U. Röthlisberger, W. Andreoni, and P. Giannozzi, J. Chem. Phys. 96, 1248 (1992).

${ }^{28}$ J. Jeong, I. Lee, J. Oh, and K. Chang, J. Phys.: Condens. Matter 10, 5851 (1998).

${ }^{29}$ M. Jarrold and E. Bower, J. Phys. Chem. 92, 5702 (1988).

${ }^{30}$ The formulas for the rotational and vibrational free energy are given in standard textbooks, see, for example, F. Jensen, Introduction to Computational Chemistry (Wiley, New York, 1999).

${ }^{31}$ F. Baletto and R. Ferrando, Rev. Mod. Phys. 77, 371 (2005).

${ }^{32}$ T. B. Coplen, J. K. Bohlke, P. De Bievre, T. Ding, N. E. Holden, J. A. Hopple, H. R. Krouse, A. Lamberty, H. S. Peiser, K. Revesz, S. E. Rieder, K. J. R. Rosman, E. Roth, P. D. P. Taylor, R. D. Vocke, Jr., and Y. K. Xiao, Pure Appl. Chem. 74, 1987 (2002).

${ }^{33}$ O. Guliamov, L. Kronik, and K. A. Jackson, J. Chem. Phys. 123, 204312 (2005). 